\title{
Recent Events
}

\section{York University's 5th Lillian Meighen Wright Maternal-Child Health Learning Institute}

Luz Maria Vazquez, Office of Women's Health Research Chair in Mental Health, York University

On November 1, 2019, our Office held the 5th Lillian and Meighen Wright Learning Institute. Keynote speaker Deborah McGregor presented on Indigenous Women, Gender-Based Violence and Decolonization. Invited panelists presented on research projects and community interventions around the two themes of the Institute: Maternal-Child Health, and GenderBased Violence and Trauma- Informed Approaches. Panelists presented on topics including Perinatal Maternal Mental Health, Black Maternal Health Experiences in Canada, Trauma-Informed Multidisciplinary Response to Child Abuse, Caesarean Section Factors Affecting Women's Preferences and Clinicians' Counsel, Health Seeking Behaviors in Canadian Immigrant Postpartum Women, GenderBased Violence Against Refugee Youth and TraumaInformed Practice, Sex Trafficking of Underage Girls: Risk Factors and Intervention, Social Organization of How To Get Out of Abusive Relationships, The Peer Champion as a Promising Practice, and TraumaInformed Shelter: From the Ground Up. The event also included poster presenters from community agencies, students and members of the academic community.

The event booklet (including abstracts of the presentations) is available at: http://nkhanlou.info.yorku.ca/files/2019/11/FinalBooklet-5th-LMW-Learning-Institute.2019.pdf

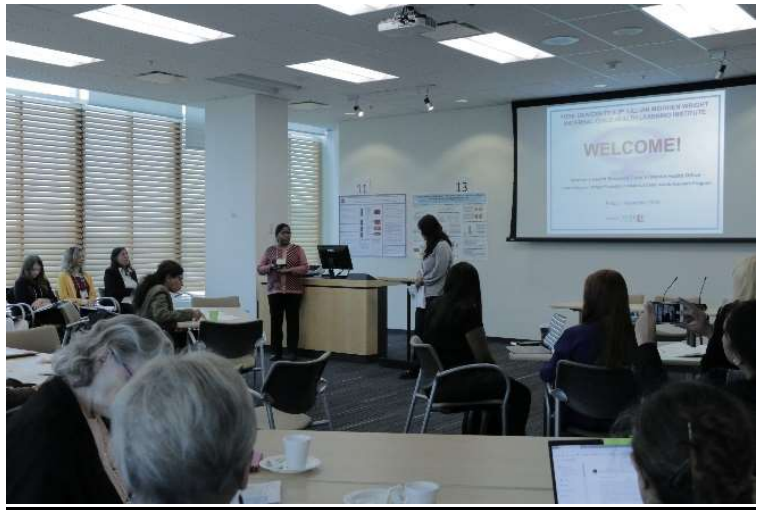

